\title{
Correction: A RasGAP, DAB2IP, regulates lipid droplet homeostasis by serving as GAP toward RAB40C
}

\section{Xiaomin Luo ${ }^{1,2}$, Chunman Li',*, Ran Tan ${ }^{3, *}$, Xiaohui Xu ${ }^{3}$, William K.K. Wu ${ }^{4}$, Ayano Satoh $^{5}$, Tuanlao Wang ${ }^{3}$ and Sidney $\mathbf{Y u}^{2,6}$}

${ }^{1}$ Guangdong and Shenzhen Key Laboratory of Male Reproductive Medicine and Genetics, Institute of Urology, Peking University Shenzhen Hospital, Shenzhen PKU-HKUST Medical Center, Shenzhen, P.R. China

${ }^{2}$ School of Biomedical Sciences, The Chinese University of Hong Kong, Hong Kong SAR, P.R. China

${ }^{3}$ School of Pharmaceutical Sciences, Xiamen University, Fujian, P.R. China

${ }^{4}$ Department of Anesthesia, Faculty of Medicine, The Chinese University of Hong Kong, Hong Kong SAR, P.R. China

${ }^{5}$ The Graduate School of Natural Science and Technology, Okayama University, Okayama, Japan

${ }^{6}$ Epithelial Cell Biology Research Centre, The Chinese University of Hong Kong, Hong Kong SAR, P.R. China

* These authors have contributed equally to this work

Published: March 02, 2018

Copyright: Luo et al. This is an open-access article distributed under the terms of the Creative Commons Attribution License 3.0 (CC BY 3.0), which permits unrestricted use, distribution, and reproduction in any medium, provided the original author and source are credited.

This article has been corrected: The proper order of the affiliations is as follows:

${ }^{3}$ School of Pharmaceutical Sciences, Xiamen University, Fujian, P.R. China

${ }^{4}$ Department of Anesthesia, Faculty of Medicine, The Chinese University of Hong Kong, Hong Kong SAR, P.R. China

Original article: Oncotarget. 2017; 8:85415-85427. https://doi.org/10.18632/oncotarget.19960 